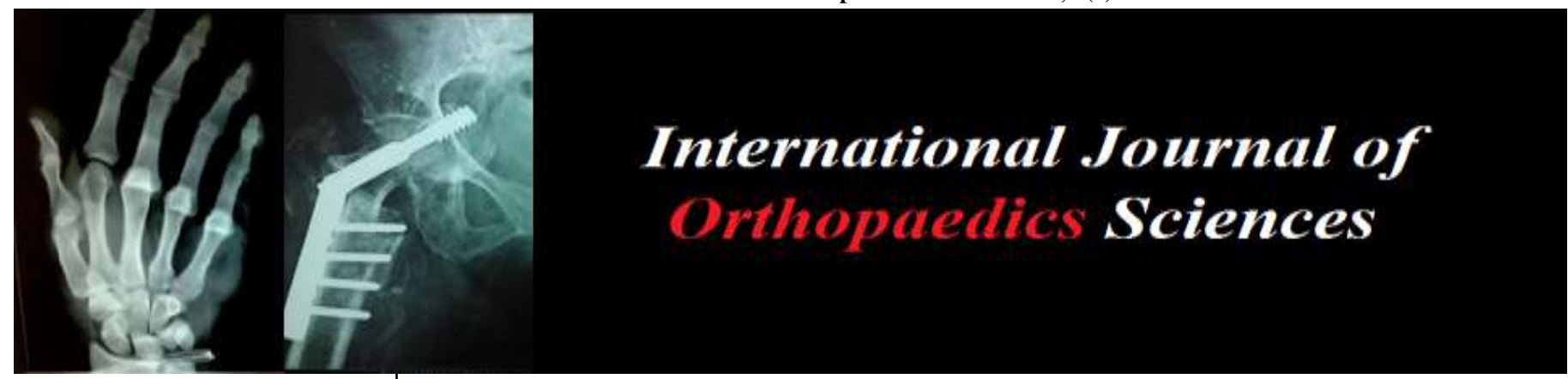

ISSN: $2395-1958$

IJOS 2018; 4(2): 226-230

(C) 2018 IJOS

www.orthopaper.com

Received: 07-02-2018

Accepted: 10-03-2018

Dr. Ashok Vidyarthi MS

Associate Professor, Department of Orthopaedic Netaji Subhash

Chandra Bose Medical College,

Jabalpur MP, India

Dr. Ashish Sirsikar, MS

Assistant Professor, Department of Orthopaedic Netaji Subhash Chandra Bose Medical College,

Jabalpur MP, India

Dr. Aashish Raghu, MS Orthopaedic Consultant Vyādhi Institute of Medical

Sciences Epip Area,

Whitefield, Bengaluru

Karnataka, India
Correspondence

Dr. Ashish Sirsikar

Assistant Professor, Department

of Orthopaedic Netaji Subhash

Chandra Bose Medical College,

Jabalpur MP, India

\section{A comparative evaluation of results of proximal femur nail and dynamic hip screw fixation for intertrochanteric femur fractures}

\author{
Dr. Ashok Vidyarthi MS, Dr. Ashish Sirsikar MS and Dr. Aashish Raghu, \\ MS
}

DOI: https://doi.org/10.22271/ortho.2018.v4.i2d.34

Abstract

Incidence of intertrochanteric femur fractures has increased significantly during the recent decades, due to the change in the demographic profile of Indian population. Conservative management of these fractures has shown higher mortality and morbidity and has largely been abandoned. Rigid Internal fixation and early mobilization is the standard method of treatment.

Objective: To verify the advantages and disadvantages of the proximal femur nail over the dynamic hip screw device and also their effect on the eventual functional outcome of the patient.

Methods: This is a retrospective and prospective comparative analytical study of 30 cases of intertrochanteric femur fractures admitted in Netaji Subhash Chandra Bose Medical College and Hospital, Jabalpur from January 2011 to November 2014. 15 cases were treated by PFN and 15 by DHS. Patients were followed up at 3 months, 6 months and 1 year. The results were compared for functional outcome by modified Harris Hip Score.

Results: In our study of 30 patients, there were 4 female and 26 male. Minimum age was 24 years, maximum 70 years with mean age of 46.1 years. Most of the cases sustained fracture by trivial trauma. $60 \%$ of the fractures were unstable. More duration of surgery, more blood loss, more number of radiation exposure, more chances of infection was seen with PFN, whereas more screw backout, more chances of varus \& valgus non-union was seen with DHS.

Conclusion: PFN had better functional outcome in patients with especially unstable fractures and DHS had better functional outcome among the stable fractures.

Keywords: Proximal femur nail, dynamic hip screw, intertrochanteric femur fracture

\section{Introduction}

Intertrochanteric fractures are one of the most common injuries sustained predominantly in patients over 60 years of age. They are 3 or 4 times more common in elderly women who are osteoporotic, in whom trivial trauma is the most common mode of injury ${ }^{[1,2]}$.

Conservative management usually results in malunion with varus and external rotation deformity resulting in a short limb gait and a high rate of mortality due to complication of recumbence and immobilization ${ }^{[1]}$.

The goal of treatment of an intertrochanteric fracture is the restoration of the patient to his or her pre-injury status as early as possible. This led to internal fixation of these fractures to increase patient comfort, facilitate nursing care, decrease hospitalization and reduce complications of prolonged recumbency ${ }^{[2]}$. it is universally agreed that the treatment of intertrochanteric fractures is stable internal fixation as early as possible ${ }^{[3]}$.

The type of implant used has an influence on fixation and can cause complications. Dynamic hip screw (DHS) is an eccentric load sharing device and a time tested procedure to manage these fractures but is associated with open reduction, loss of fracture hematoma, periosteal stripping and extensive soft tissue dissection. Closed Proximal Femur Nail (PFN) overcomes these shortcomings associated with DHS. Its biomechanical properties like being an axial load bearing device with a short lever arm, greater implant length, smaller and flexible distal ends and an additional derotational screw in femur neck may offer significant advantage over DHS ${ }^{[4]}$. 
The purpose of the present study is to verify the advantages and disadvantages of the proximal femur nail over the dynamic hip screw device and also their effect on the eventual functional outcome of the patient.

\section{Materials and Methods}

This is a retrospective and prospective comparative analytical study conducted in Netaji Subhash Chandra Bose Medical College and Hospital, Jabalpur, after taking clearance of ethical committee, during the period from January 2011 to November 2014. A total of 98 patients of intertrochanteric fractures of the femur were operated during this period in our institute. Among these patients, 26 patients died after surgery and were not included in this study. Some of the patients were not traceable via telephone and other means. Patients who were followed up for upto 1 year were included in this study.

\subsection{Inclusion criteria}

Patients aged above 18 years; Patients with isolated intertrochanteric fractures types 31 A1, A2, A3; Patients who were ambulatory before the injury; Delayed union/ Nonunion/ Failed intertrochanteric fractures.

\subsection{Exclusion criteria}

Patients younger than 18 years of age; Trochanteric fractures associated with any other fracture like neck or shaft of femur; Compound fractures; Pathological fractures: Primary malignancy / metastatic disease, Osteomyelitis of the proximal femur, Bone marrow disease such as myelodysplastic syndrome, Metabolic bone disorders other than osteoporosis like Osteomalacia, Renal osteodystrophy; Peri-prosthetic fractures; Patients who were non-ambulatory before the injury; Patients not giving consent for the surgery. The patients were evaluated as per the history, mode of injury, clinical examination, necessary radiological investigations and hematology profile which was done on admission. Fracture was classified as per AO/OTA Classification. All patients had similar antibiotic coverage, and spinal or epidural anaesthesia as decided by the anaesthetist was given. Closed reduction was done under $\mathrm{C}$-arm image guidance and the fracture was fixed by either PFN or DHS by the same surgeon.

Postoperatively, all patients were given Intravenous antibiotics Ceftriaxone-Sulbactam combination 1.5 for Gram positive and Gram negative coverage for 48 hours. The immediate post-operative X-rays were evaluated. Patients were allowed to sit up in bed on the second post-operative day. Isometric quadriceps and Ankle pump exercises where started on the second and third post-operative day. Sutures were removed after 12 days. Patients were mobilized on Nonweight bearing by hanging the legs on the side of the bed and encouraging knee flexion and extension exercises as soon as the pain or general condition permitted. Weight bearing was commenced depending upon the stability of the fracture and adequacy of fixation, delaying it for patients with unstable or inadequate fixation.

All the patients were followed up at 3 months, 6 months and 1 year, and check $\mathrm{x}$ - rays were taken to assess fracture union and signs of failure of fixation. Post-operative walking ability of each patient was recorded and compared with pre-injury walking ability using the Sahl strand grading. Post-operative pain was evaluated using the pain criteria provided in Modified Harris Hip Score ${ }^{[5]}$.

\section{Results}

In the present study, 30 Cases were selected. Maximum numbers of the Cases were seen in 51-70 years age group (47.5\%). 1 female (6.7\%) and 14 males (93.3\%) with intertrochanteric femur fractures were treated with DHS and 3 females (20\%) and 12 males $(80 \%)$ were treated with PFN. Majority of the Cases were trivial trauma of fall on ground 10 Cases $(33.33 \%)$ followed by fall from height 8 Cases (26.66\%) followed by road traffic accident in 6 Cases $(20 \%)$. Majority were unstable fractures (A2.2, A2.3, A3.1, A3.2, A3.3) 18 Cases (60\%) followed by stable fractures (A1.1, A1.2, A1.3, A2.1) 12 Cases (40\%). In patients of age group $<30$ to 60 years, the majority sustained high energy trauma by fall from height or Road Traffic Accident (RTA) because of active lifestyle and more mobility than old age group. In patients of age group $>60$ years, trivial trauma is the dominant mode of injury, which can be attributed to the less active lifestyle, lesser mobility among old age persons and also osteoporotic bone quality which makes them more prone for fractures even from trivial trauma.

In patients aged less than 50 years, most of them sustained upto Type A2.3 fractures but as the Age group increases from here, extension to more complex and unstable fracture variants were seen. There were 12 stable fractures and 18 unstable fractures (A2.2 and above) out of which, 6 unstable fractures were treated by DHS and 12 unstable fractures were treated by PFN. The reason for this can be attributed to the surgeon's choice of implant according to his familiarity, comfort and confidence, possible misjudgment of the fracture stability according to the AO/OTA Classification.

The mean length of incision was longer in PFN $9.0 \mathrm{~cm}$ [Not Statistically significant]. The duration of surgery was not significantly different in both groups $(\mathrm{p}=0.21)$. More time was taken in performing PFN than in DHS. The increase blood loss in DHS is attributed to longer incision and more soft tissue handling as compared to PFN. The mean no. of radiation exposure with $\mathrm{C}$-Arm shoots was more in PFN, probably because of steeper learning curve of the surgeons performing this procedure, hence more time needed in correct placement of the nail and screws. Chronic infection was seen in the PFN operated group which can be attributed to longer duration of surgery in PFN. Backing out of the screw in DHS was seen in 4 Cases of the 15 DHS operated patients and backing out of $8 \mathrm{~mm}$ hip screw was seen in 1 Case in PFN operated patient.

From total of 15 patients each of DHS and PFN, 4 patients from DHS operated group showed Varus malunion and 1 patient from DHS group showed Valgus malunion and 1 patient from the PFN group showed Varus malunion and rest of the patients showed union in normal range. All patients were in Sahlstrand's Grade 1 walking ability before trauma. Post-op walking ability in this study shows $60 \%$ PFN patients walking without support with $33.3 \%$ DHS patients walking without support and $66.7 \%$ patients of DHS walking with cane and $13.3 \%$ patients of PFN needing 2 canes or walker post-operatively measured at 6 months post-op.

With increasing complexity of the fracture pattern from A2 to A3, the No. of C-Arm Image intensifier shoots were also found to increase. This can be attributed to more time taken for adequate reduction and fixation of complex unstable fractures when compared to more simple and stable fracture patterns. In patients treated with DHS, it was seen that as fracture complexity increases, the shortening also increased. This can be attributed to the fact that there is more fracture collapse in fractures with deficient lateral walls as seen in the 
more complex types.

Harris Hip score was found to be more in patients treated with PFN. Upto Age group 60 years, the patients have increasing Harris Hip scores but ages more than 60 years onwards the Harris Hip score values are depreciating. This could be attributed to various factors like post-op rehabilitation, activity level, motivation to perform active tasks, muscle strength, fracture consolidation which can all be affected by the age.

As complexity of the fracture pattern increases, the Harris Hip score measurement was found to be in lower score values. This can be attributed to the decrease in stability in more complex fractures which has a bearing on the fracture consolidation, pain at fracture site, and more period of nonfull weight bearing on the limb which can indirectly cause reduced muscle strength.

\section{Discussion}

In our present study, out of the 30 cases that were selected, maximum numbers of the cases were seen in 50-70 years age group (30\%) and also in 30-40 years age group (30\%). The majority of 50-70 years age group sustained trivial trauma by fall on ground, and the 31-40 years age group sustained high energy trauma by fall from height or road traffic accident (RTA) because of active lifestyle and more mobility than old age group. White and colleagues did a study of prevalence of intertrochanteric fractures in elderly patients and concluded that the average age for trochanteric fractures is 75.4 years. ${ }^{6}$ Intertrochanteric fractures show a bimodal distribution (20 to 40 years and over 60 years) according to a study by Brunner

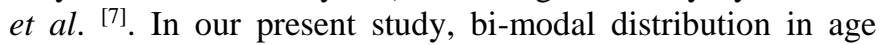
groups was seen similarly as in the study conducted by Brunner et al. ${ }^{[7]}$.

In our study, $86.6 \%$ patients were male and $13.3 \%$ were female. Dahl and colleagues ${ }^{[8]}$, in their study concluded $65 \%$ of female patients, explained by the fact that female are more prone for the osteoporosis after menopause. The highest rates were found among women, occurring in a 3:1 female to male ratio according to study by David G. Lovelle et al. ${ }^{\text {[9] Our }}$ observations do not match with the various literature and studies published which could be due to possible sex discrimination in our region with less care and somewhat negligent attitude offered by the family members to elderly female persons.

In our study, majority were Trivial trauma of fall on ground $(33.33 \%)$ followed by High energy trauma of fall from height (26.66\%), followed by RTA (20\%). This matches with various other studies like those by Waters and Millers et al. [10] in 2002, supporting the view that bone stock plays an important role in fracture proneness, where higher the trauma force seen in younger and active individuals and trivial trauma causing fracture in less active, elderly persons with poorer bone stock.

In our study, $60 \%$ of the patients had unstable type intertrochanteric fractures. The incidence of osteoporosis in patients with unstable fracture was $66.67 \%$ (50\% of patients were with type A2.2 and type A2.3), whereas $1.7 \%$ patients with a stable AO A1 fracture were found in the study conducted by Deepak Joshi and Anoop C in November 2014 ${ }^{[11]}$. Other studies also indicate higher fracture comminution among persons with poor bone stock and higher energy trauma. Our results match with these studies.

In the present study, mean of $9.0 \mathrm{~cm}$ long incision was seen in PFN with only $8.8 \mathrm{~cm}$ mean among patients treated with DHS. This is comparable to the study conducted by Zhao C et al. ${ }^{[3]}$ in $2009(9.6 \pm 0.9) \mathrm{cm}$ in PFN; $(15.5 \pm 1.5) \mathrm{cm}$ in DHS and $5 \mathrm{~cm}$ in study conducted by Nargesh et al. ${ }^{[12]}$ in 2013 where incision in $P F N$ was $8.5 \pm 1.2 \mathrm{~cm}$ while in $D H S$, the length of incision was $16 \mathrm{~cm}$. This can be attributed to the fact that separate incision was required for entry point for the nail and separate incisions for the hip screw and locking bolts. In DHS usually single incision to allow the passage of side plate after fixation with Richard's screw, which requires quite meticulous exposure. In our study, the combined length of the separate incisions for PFN was found to be more than the single incision for DHS.

In our study, more time was taken in performing PFN (Mean 2.5 hours) than DHS (1.7 hours) because it is a closed procedure needing frequent $\mathrm{C}$-Arm image views to confirm reduction and position of implants separately for the guide wire, nail, $8 \mathrm{~mm}$ screw and $6.5 \mathrm{~mm}$ screw in performing PFN rather than in DHS, which require C-Arm much less because of more visualization in open procedure. Baumgaertner et al. ${ }^{[13]}$ found that the surgical times were $10 \%$ higher in the DHS group in their series. Saudan and colleagues ${ }^{[14]}$ found that there was no significant difference between the operative times in the two groups in their series. Zhao $\mathrm{C}$ et al. ${ }^{[3]}$ found an average time of operation was $(51.5 \pm 4.4) \mathrm{min}$ in PFN; $(68.8 \pm 5.9) \mathrm{min}$ in DHS which was due to performing surgeons being more experienced in performing PFN. Our study does not match most of the above studies because PFN procedure requires long frequent $\mathrm{C}$-Arm exposure to confirm the position of the implants in each step and also more time was required as optimal operating instruments like electric cannulated reamer/drill was not available in our institute during the study period which made manual drilling and reaming of the hip screws more difficult and time consuming. In our study, more no. of C-Arm Image Intensifier shoots were taken in PFN (Mean 50.3 shoots, which has statistically significant difference with Mean 15.9 shoots in DHS) because of more time needed in correct placement of the nail and screws as it is a closed procedure, which would be more than in DHS and also steep learning curve needed for this procedure and intra-op confidence of the surgeon regarding near anatomical reduction and stable fixation being performed in closed manner during PFN insertion. This was similar to the series by Baumgaertner and associates ${ }^{13}$ who also found a significant difference in the fluoroscopic times in their series, with $10 \%$ higher times for the PFN group. However in their study, Saudan et al. ${ }^{[14]}$ found no difference between the fluoroscopy times in both the groups which can be attributed to more experienced surgeons performing the respective procedure and in less complex fracture configurations in their study.

In the present study, the mean blood loss during DHS $(250.67 \mathrm{ml})$ was significantly more than that during PFN $(202.7 \mathrm{ml})$. In a study by Zhao C et al. ${ }^{[3]}$ the blood loss was $(179.0 \pm 12.9) \mathrm{ml}$ in PFN; $(269.3 \pm 40.0) \mathrm{ml}$ in DHS. Pan et al. [15] in 2004 and Pajarinen et al. ${ }^{[16]}$ in 2005 concluded similarly in this regard. Our observation matches with those in the above studies. This can be attributed to the longer incision and more soft tissue dissection and handling while performing DHS which leads to more blood loss than in PFN which requires relatively smaller incisions and less soft tissue handling.

Results of treatment of stable and unstable fracture have usually been reported together in the literature, and it is generally accepted that with increasing complexity of fracture pattern (stable to unstable), there is a higher risk of complication and poor outcome. The only complications 
encountered in the present study were malunion, screw backout and wound infection. Post-operative complications Chronic wound infection was seen in 2 Cases of PFN operated patients. 4 cases of DHS operated patients had Richard's screw backout, 1 Case of $8 \mathrm{~mm}$ hip screw backout in PFN operated patient. In the present study, shortening of the limb was significantly $(\mathrm{p}=0.01)$ more in DHS operated patients (Mean $1.8 \mathrm{~cm}$ ) than PFN operated patients (Mean $1.1 \mathrm{~cm})$.

The wound infection in PFN could be attributed to the longer time taken for the surgery, which can expose the incision to micro-organisms for longer time, sometimes frequent revision of the hip screws due to unsatisfactory fixation discerned by the operating surgeon on table which can put higher risk of the wound to infection and difficulty in washing smaller incision wounds than larger wounds.

In the present study, Varus malunion was seen in 4 cases of DHS associated with Richard's screw backout and 1 case in PFN operated patients each and single case of valgus malunion was seen in DHS operated patient. One reason may be that the entry point of the PFN at the tip of the greater trochanter is located directly in the fracture region which can cause an intraoperative fracture displacement and varus angulation. In DHS operated patients, varus malunion is frequent due to medialisation of the distal fragment during excessive collapse of the lateral wall in case of unstable fractures. Screw backout was seen in a 4 cases of DHS associated with varus malunion and $2-2.5 \mathrm{~cm}$ shortening due to excessive fracture collapse in unstable fractures A2,2 and A2.3. Single case distal screw backout was seen in PFN because of the lack of maintaining proper Tip Apex Distance between the tip of the hip screw and the joint and positioning further away from the inferior border of the neck and the postero-medial cortex.

In the present study, patients operated with DHS exhibited higher pain grades according to the section in Modified Harris Hip Score at 6 months, as compared to PFN due to increased fracture collapse especially in lateral wall deficient fractures and malunion into varus or valgus associated with shortening. $46.7 \%$ of DHS patients had slight pain with no compromise in activity and $13.3 \%$ of DHS patients had mild pain with no effect on ordinary activity but some pain on performing unusual activity, post-operatively at 6 months. This can be attributed to higher fracture collapse in unstable fractures especially those with deficient lateral walls. In PFN, the protruding hip screws and proximal tip of nail were the main cause of pain for the patients. Saudan and colleagues [14] found that the amount of persistent pain was similar in both groups in their series.

On taking total of $300^{\circ}$ hip range of movements according to the criteria in Modified Harris Hip Score at 6 months follow up, PFN operated patients (Mean $152.7^{\circ} / 300^{\circ}$ ) had better range of movement than DHS operated patients (Mean $141.7 \%$ ). This is comparable to the results put forth by Saudan and colleagues ${ }^{[14]}$ and also the study conducted by Shahid Syed Noor ${ }^{[15]}$ in 2010. This can be attributed to earlier mobilization, weight bearing in PFN operated patients as compared to the DHS operated patients since PFN is load sharing device as compared to DHS being a load bearing device and also closer proximity of the PFN implant to the vertical axis of the body thus reducing the energy expenditure in movement. In DHS, patients with unstable fractures were delayed mobilization and weight bearing but in PFN, the weight bearing was started earlier due to the load sharing implant characteristics of PFN.
In the present study, shortening of the limb was significantly $(\mathrm{p}=0.01)$ more in DHS operated patients (Mean $1.8 \mathrm{~cm})$ than PFN operated patients (Mean $1.1 \mathrm{~cm}$ ). This could be attributed to the fact that in DHS, controlled fracture collapse occurs over a sliding implant. If the collapse is more than $15 \mathrm{~mm}$ (GS Kulkarni [18] 2006) there is higher chance of excessive shortening and implant failure. The 4 patients with Varus malunion had excessive shortening.

In the present study, the Functional outcome was taken according to the Modified Harris Hip Score and was found to be better for PFN treated patients (Mean score 76.8) than for DHS treated patients (Mean score 75.6), but was not statistically significant $(\mathrm{p}=0.74)$. Excellent Functional outcome was seen in two DHS operated patients (13.3\%) and in one PFN operated patient $(6.7 \%)$, Good Functional outcome $(33.3 \%)$ in PFN operated patients, Fair functional outcome $53.3 \%$ among DHS operated group.

On taking Age vs Functional outcome, upto age group 60 years, the patients have increasing Harris Hip scores but ages more than 60 years onwards the Harris Hip score values are depreciating. This could be attributed to various factors like post-op rehabilitation, activity level, motivation to perform active tasks, muscle strength, fracture consolidation which can all be affected by the age. Similar findings were seen in the series by Pajarinen and group ${ }^{16}$. This suggests that the use of PFN may be favored in stable fracture when compared to DHS. In the study by Xiao Huang ${ }^{19}$ in 2009 , there was no difference in the functional outcome between DHS and PFN. On taking Type of fracture vs Functional outcome, as complexity of the fracture pattern increases, the Harris Hip score measurement was found to be in lower score values. This can be attributed to the decrease in stability in more complex fractures which has a bearing on the fracture consolidation, pain at fracture site, more period of non-full weight bearing on the limb which can indirectly cause reduced muscle strength. Similar results were observed in Jaswinder Pal Singh et. Al. ${ }^{20}$ study of Stable and Unstable Intertrochanteric fractures treated by PFN and DHS in 2007. In addition, with unstable intertrochanteric fractures, the PFN has a definite advantage over the DHS in terms of less limb length shortening, earlier restoration of pre-injury walking ability and a better overall functional outcome. In the present study, the pros of PFN surgery includes lesser blood loss, less shortening, earlier weight bearing, lesser union complications but more exposure of the surgeon and patient to fluoroscopy radiographic exposure intra-op and longer duration of surgery. DHS offered relatively easier method to the surgeon, which can be mastered at relatively shorter learning curve, measured by the lesser time taken to perform the surgery and lesser radiographic exposure than PFN but not very effective in more unstable fracture patterns as it tended to cause more shortening in unstable fractures associated with varus malunion as well, associated with shortening.

\section{Conclusion}

In our study, PFN was compared with DHS for the operative treatment of intertrochanteric fractures in terms of peroperative and post-operative variables. It was found that PFN had better functional outcome in patients with especially unstable fractures and DHS had better functional outcome among the stable fractures.

Both PFN and DHS have their advantages and disadvantages in treatment of intertrochanteric fractures. There are various variables which need to be considered in order to decide which patient should be treated with which kind of implant 
which is left to the safe discretion of the operating surgeon by his expertise, knowledge and experience.

\section{References}

1. Chang WS, Zuckerman JD, Kummer FJ, Frankel VH. Biomechanical evaluat ion of anatomic reduction $\mathrm{v} / \mathrm{s}$ medial displacement osteotomy in unstable intertrochanteric fractures. $\mathrm{Cl}$ in Orthop. 1998; 225:145-6.

2. NK Karn, Ashish Jain, Mahi Pal Singh. A Prospective Randomized Control Trail Comparing Proximal Femoral Nail and Sliding Hip Screw in The Management of Trochanteric Fracture of The Femur. Health Renaissance. 2011; 9:7-11.

3. Zhao C, Liu DY, Guo J, et al. Comparison of Proximal femoral nail and dynamic hip screw for treating Intertrochanteric fractures. China journal of Orthopaedics and traumatology. 2009; 22(7):535537.

4. Evans EM. The treatment of trochanteric fractures of the femur. J Bone Joint Surg. 1949; 31B:190-203.

5. Harris WH. Traumatic arthritis of the hip after dislocation and acetabular fractures: treatment by mold arthroplasty. An end-result study using a new method of result evaluation. J Bone Joint Surg Am. 1969; 51:737-55.

6. White BL, Fisher WD, Laurin CA. Rate of mortality for elderly patients after fracture of the hip in the 1980's. J Bone Joint Surg. 1987; 69A:1335-40.

7. Brunner LC, Eshilian-Oates L, Kuo TY. Hip fractures in adults. Am Fam Physician 2003; 67:537.

8. Dahl E. Mortality and life expectancy after hip fractures. Acta Orthop Scand. 1980; 51:163-70.

9. Dean GL, David S, Jason HN. Osteoporotic pertrochanteric; management and concurrent controversies. J Bone Jt Surg (Am) 72-B, 737-752.

10. Prediction of fracture risk in postmenopausal white women with peripheral bone densitometry: evidence from the National Osteoporosis Risk Assessment. Miller PD, Siris ES, Barrett-Connor E, Faulkner KG, Wehren LE, Abbott TA, Chen YT, Berger ML, Santora AC, Sherwood LM. J Bone Miner Res. 2002; 17(12):2222-30.

11. Joshi D, Dhamangaonkar AC, Ramawat S, Goregaonkar AB. Predictors of iatrogenic lateral wall fractures while treating intertrochanteric fracture femur with dynamic hip screw system in Indian patients. Eur J Orthop Surg Traumatol 2015; 25:677-82.

12. Nargesh A et al. Comparative study of the management of inter-trochanteric fractures in the elderly: short proximal femoral nail vs dynamic hip screw. Sri Lanka Journal of Surgery. 2013; 30(2):1317. DOI:http://doi.org/10.4038/sljs.v30i2.5176

13. Baumgaertner MR, Curt in SL, Lindskog DM. Intramedullary versus extramedullary fixation for treatment of intertrochanteric hip fractures. ClinOrthop. 1998; 348:87-94.

14. Saudan M, Lubbeke A, Sadowski C, Riand N, and Stern R, Hoffmeyer P. Pertrochanteric fractures: is there an advantage to an intramedullary nail? A randomized, prospect ive study of 206 patients comparing the dynamic hip screw and proximal femoral nail. J Orthop Trauma. 2002; 16:386-393.

15. Minos Tyllianakis, Andreas Panagopoulos, and
Andreas Papadopoulos, et al. Treatment of extracapsular hip fractures with the proximal femoral nail (PFN): Long term results in 45 patients. Acta Orthop.Belg. 2004; 70:444-454.

16. J Pajarinen, J Lindahl, O Michelsson, V Savolainen, E Hirvensalo, Pertrochanteric femoral fractures treated with a dynamic hip screw or a proximal femoral nail: a randomised study comparing postoperative rehabilitation, Journal of Bone and Joint Surgery B. 2005; 87(1):76-81,

17. Syed Shahid Noor, Niaz Hussain, Muhammad Tariq Karim, Imran Gabol. Outcome of Dynamic Hip Screw in patients with Intertrochanteris femur fractures. The Journal of Pakistan Orthopaedic Association. Feb. 2011; 23(1):40-43.

18. GS Kulkarni, Rajiv Limaye, Milind Kulkarni, Sunil Kulkarni. Post Graduate Institute of SwasthiyogPratishthan, Extn. Area, Miraj, Maharashtra; Current Concept review; Intertrochanteric fractures; Indian Journal of Orthopaedics. 2006; 40:(1):16-23.

19. Xiao Huang, Frankie Leung, Zhou Xiang, Pei-Yong Tan, Jing Yang, Dai-Qing Wei.et al. Femoral Nail versus Dynamic Hip Screw Fixation for Trochanteric Fractures: A Meta-Analysis of Randomized Controlled Trials. The Scientific World Journal Article ID 805805, 2013, 8.

20. Jaswinder Pal Singh Walia, Himanshu Tailor, HS Mann, Avinash Chander Gupta, Jagdeep Singh Rehncy, Sargun Singh. A Comparative study of 30 cases of trochanteric fracture femur treated with dynamic hip screw and proximal femoral nailing. $\mathrm{Pb}$ Journal of Orthopaedics Vol-2013; 15(1). 